\title{
Evolution of Magnetic Fields in Galaxies
}

\author{
J. M. Stil* \\ Institute for Space Imaging Science \\ Department of Physics and Astronomy \\ The University of Calgary \\ E-mail: stil@ras.ucalgary.ca
}

Faraday rotation of polarized radio sources is one of the most effective observational tools to study cosmic magnetic fields. A densely-sampled grid of Faraday rotation measures that covers the complete sky (the Rotation Measure Grid) will allow us to probe the magnetic field in the Milky Way Galaxy as well as in other nearby galaxies. First results from a new database of 37543 rotation measures that covers over $80 \%$ of the sky show the potential of an all-sky polarization survey with SKA path finders and the SKA. Structures in RM on angular scales of tens of degrees and systematic variation in the amplitude and slope of rotation measure structure functions across the sky are presented. A future all-sky RM grid of $\sim 5000$ per square degree will allow detailed mapping of the magneto-ionic medium, and also probe $\sim 1000$ nearby galaxies with at least 20 rotation measures per galaxy. Observations of magnetic field structure in more distant galaxies will rely on the detection of polarized synchrotron emission for which a deep field would have the necessary sensitivity. At high redshift, imaging the polarized structure of normal galaxies at a resolution of $\sim 0.1^{\prime \prime}$ or better requires sensitivity beyond the design specifications of the SKA. However, the polarization of unresolved disk galaxies as a function of redshift provides a way to probe the evolution of magnetic fields in disk galaxies over time.

Panoramic Radio Astronomy: Wide-field 1-2 GHz research on galaxy evolution - PRA2009

June 02 - 052009

Groningen, the Netherlands

\footnotetext{
* Speaker.
} 


\section{The RM Grid}

Magnetic fields are an essential part of many astrophysical phenomena, but fundamental questions remain about their evolution, structure, and origin. Polarization of radio synchrotron emission and Faraday rotation have been successful tools to probe large-scale magnetic fields in the Milky Way (e.g. [1, 2, 3] and references therein), and other galaxies (e.g. [4, 5])

Magnetic fields on small angular scales can be revealed by rotation measures (RMs) of polarized background sources if the density of these sources is high enough to probe a single structure with multiple lines of sight. The sensitivity to foreground RM and the angular resolution both depend on the density of polarized background sources that is determined by the sensitivity of the RM survey and the polarized radio source counts. Future deep polarization surveys with the SKA prompted the extrapolation of polarized radio source counts to predict the density of a "RM grid" made with a survey of given sensitivity [8]. Later extrapolations were based on a power law extrapolation [9] of faint polarized radio source counts by [10], and by fitting polarized radio source counts with models of radio source populations that include polarization [11, 12]. These extrapolations are are made over as much as 4 orders of magnitude in polarized flux density (from $\sim 1$ mJy to $\sim 0.1 \mu \mathrm{Jy}$ ), with a high level of uncertainty. The predicted density of RMs on the sky is therefore a significant source of uncertainty in the design of polarization surveys with SKA path finders and the SKA.

\section{RM structures of the (local) ISM}

Figure 1 shows 37543 RMs derived from the NVSS [13] overlayed on all-sky images of the intensity of $\mathrm{H} \alpha$ emission [14] (top) and diffuse soft X-ray emission [18] (bottom). The RM data contain as imprint of the large-scale Galactic magnetic field that reverses sign with respect to the Galactic equator [13], but we will limit the discussion to structures on smaller angular scales. Some HII regions are associated with high $|\mathrm{RM}|$ values [15], and this can be seen in Figure 1 towards the Gum nebula $\left(l \approx-108^{\circ},|b|<20^{\circ}\right)$, the $\zeta$ Oph HII region at $(l, b)=\left(+6^{\circ},+23^{\circ}\right)$, and the $\lambda$ Ori HII region at $(l, b)=\left(-165^{\circ},-12^{\circ}\right)$.

Two large regions of enhanced RM amplitude without bright $\mathrm{H} \alpha$ emission are region $\mathrm{A}^{\prime}$ bounded by $80^{\circ}<l<150^{\circ}-40^{\circ}<b<-20^{\circ}$ (approximately region A in [7]), and region $\mathrm{C}^{\prime}$ bounded approximately by $33^{\circ}<l<68^{\circ}, 10^{\circ}<b<35^{\circ}$ (part of region $\mathrm{C}$ in [7]. Rotation measures in region $\mathrm{A}$ and region $\mathrm{C}$ have opposite signs, and the sharp boundary between these regions is the Galactic plane. We do not see a null in RM amplitude at this boundary, confirming the earlier conclusion of [ [ ]. Away from the Galactic plane, we identify several locations where RM changes sign along narrow boundaries where the RM amplitude vanishes. One such boundary forms the high-longitude boundary of region $\mathrm{A}^{\prime}$. The region of positive RM was noticed in some previous RM images of the sky [7, 19]. The boundary between these regions where RM amplitude vanishes is marked by filamentary structure in $\mathrm{H} \alpha$ and coincides with the edge of Radio Loop II.

Figure 1 (bottom) shows that Region $\mathrm{C}^{\prime}$ coincides with a dark area in the diffuse X-ray sky believed to be the silhouette of a cloud that absorbs X-ray emission from the more distant Galactic halo [18]. The cloud itself is identified in HI emission at $V_{\mathrm{LSR}} \approx-2 \mathrm{~km} \mathrm{~s}^{-1}$ and it appears asso- 

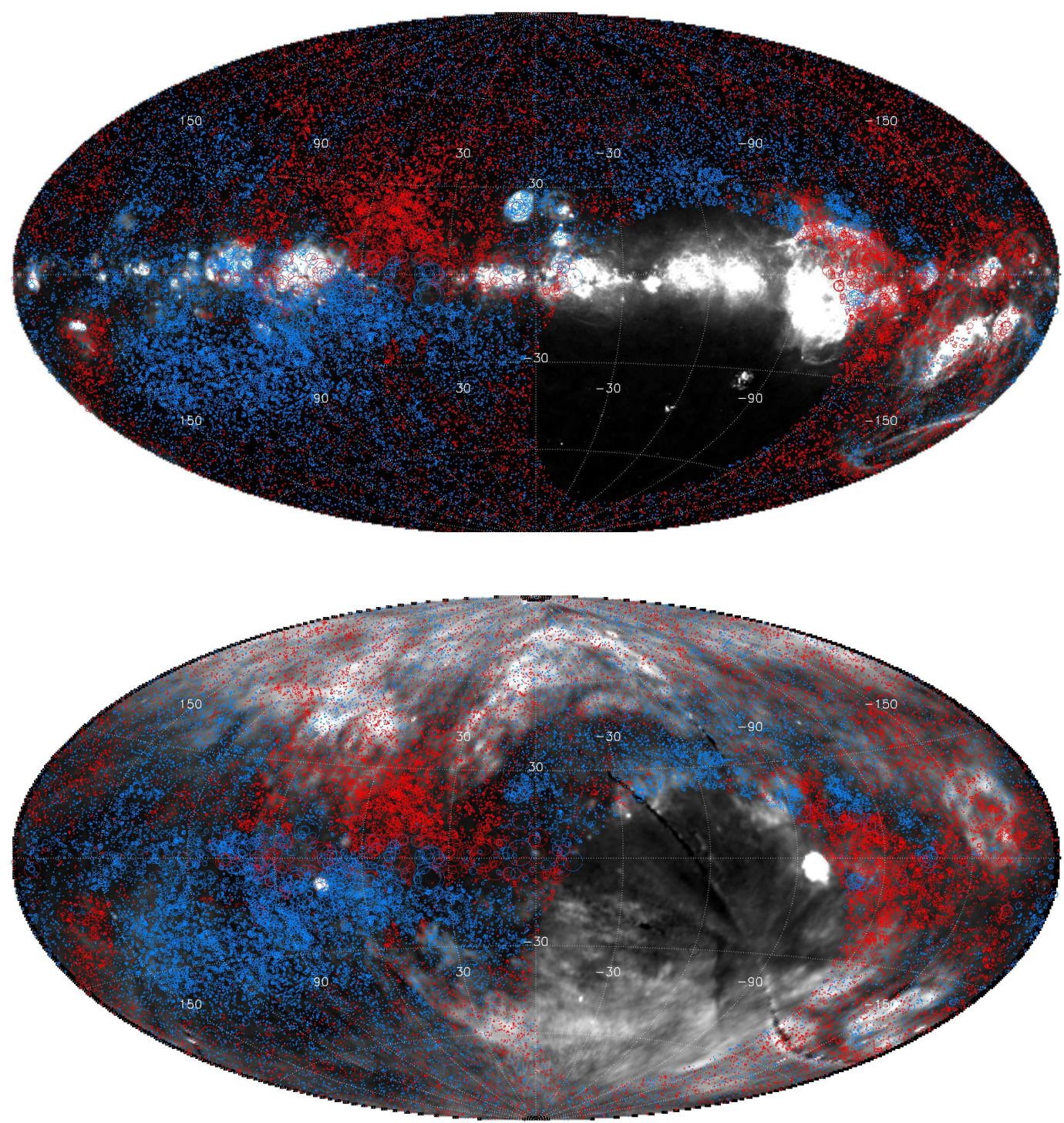

Figure 1: Top: Rotation measures from [13] on an all-sky $\mathrm{H} \alpha$ image from [14]. Red indicates positive RM, blue indicates negative RM. The symbol sizes indicate the magnitude of RM. Bottom: The same RM data set as in the top panel shown on the diffuse $0.25 \mathrm{keV}$ X-ray emission [18] measured by the ROSAT all-sky survey.

ciated with the outer HI shell of loop I. This association that local ISM structure has a measurable effect on RMs at intermediate Galactic latitude.

\subsection{RM structure functions}

RM structure on angular scales of several degrees are not sampled with enough background sources to be visible in Figure 1. Yet there appears some clustering of RMs with similar sign on angular scales of several degrees, most clearly visible at high Galactic latitudes. Better sampling by future RM surveys may reveal structure on small angular scales with much greater statistical significance and allow them to be associated with specific astronomical objects. With the present 
data we can study RM structure on small angular scales statistically by means of structure functions [20, 21, 22] $D(\delta \theta)$ defined as

$$
D(\delta \theta)=\frac{1}{N} \sum_{i}[R M(\theta)-R M(\theta+\delta \theta)]_{i}^{2},
$$

where $\delta \theta$ is the angular separation of a pair of sources for which we have RMs, and the sum is over all $N$ pairs of sources in a specified area with angular separation in a narrow range around $\delta \theta$. The units of $D$ are $\operatorname{rad}^{2} \mathrm{~m}^{-4}$. Our current data set is not sensitive to $|R M|>450 \mathrm{rad} \mathrm{m}^{-2}$, but this should not be a problem outside the Galactic plane where incidental high RMs are most likely due to sources with high internal Faraday rotation.

Figure 2 shows examples of structure functions for a few representative areas of $\sim 200$ square degrees. These graphs show the total variance of RM as a function of angular scale, not yet corrected for power added by measurement errors (noise) or variance in RM intrinsic to the extragalactic sources. These corrections will be discussed elsewhere [23]. Consistent with earlier results, e.g. [20, 21, 22, 24, 25, 28, 27, 28, we find structure function shapes that vary from flat to a rising power law, to a broken power law.

Figure 3 shows results of fitting separate power laws to structure functions of angular scales $\delta \theta<1^{\circ}$ (blue symbols) and $\delta \theta>1^{\circ}$ (red symbols). The fits for larger angular scales are much better constrained, so we adopt the normalization of the power law at $\delta \theta=1^{\circ}$ derived from the fit to $\delta \theta>1^{\circ}\left(A_{2}\right)$ as the amplitude of the SF for an angular scale of $1^{\circ}$. We see s systematic increase of both the amplitude and the slope of the SF towards the Galactic plane, with considerable symmetry between North and South. Many structure functions show a significantly steeper slope on small angular scales, although we find slopes consistent with zero for $\delta \theta<1^{\circ}$ at all latitudes. All Galactic latitudes including the Galactic polar areas show significant spread in SF amplitude. The SF in the North Galactic Pole (NGP) area also rises significantly on small angular scales. Lines of sight toward the Galactic centre show higher SF amplitudes than lines of sight towards the Galactic anticentre. This is not surprising as lines of sight at low longitude intersect much more of the Galaxy than lines of sight towards the anticentre. The most conspicuous variation in the slope of the SF is that the steepest slopes for $\delta \theta>1^{\circ}$ occur in the direction of the Gum nebula and the $\zeta$ Oph HII region.

\section{External galaxies}

Extragalactic work on magnetic fields also benefits greatly from a high sampling density of RM. Besides the Milky Way, the only galaxies so far that have been probed with a sample of RMs that can reveal the large-scale structure of the magnetic field are M31 [29], the LMC [30], and the SMC [31], thanks to their large angular size. Rotation measures are sensitive to the direction of the line of sight component of the large-scale magnetic field, allowing the detection of magnetic field reversals in galaxies, and patterns predicted by certain dynamo modes [9]. Figure 4 shows the number of galaxies as a function of solid angle derived from the UGC [32]. The solid angle $\Omega$ is defined here as the area within twice the optical radius listed in the UGC. The dashed curve in Figure $\bigoplus$ indicates spiral galaxies only, while the solid curve includes all galaxy types, including irregular and elliptical galaxies. The vertical lines in Figure 4 indicate solid angles of galaxies 

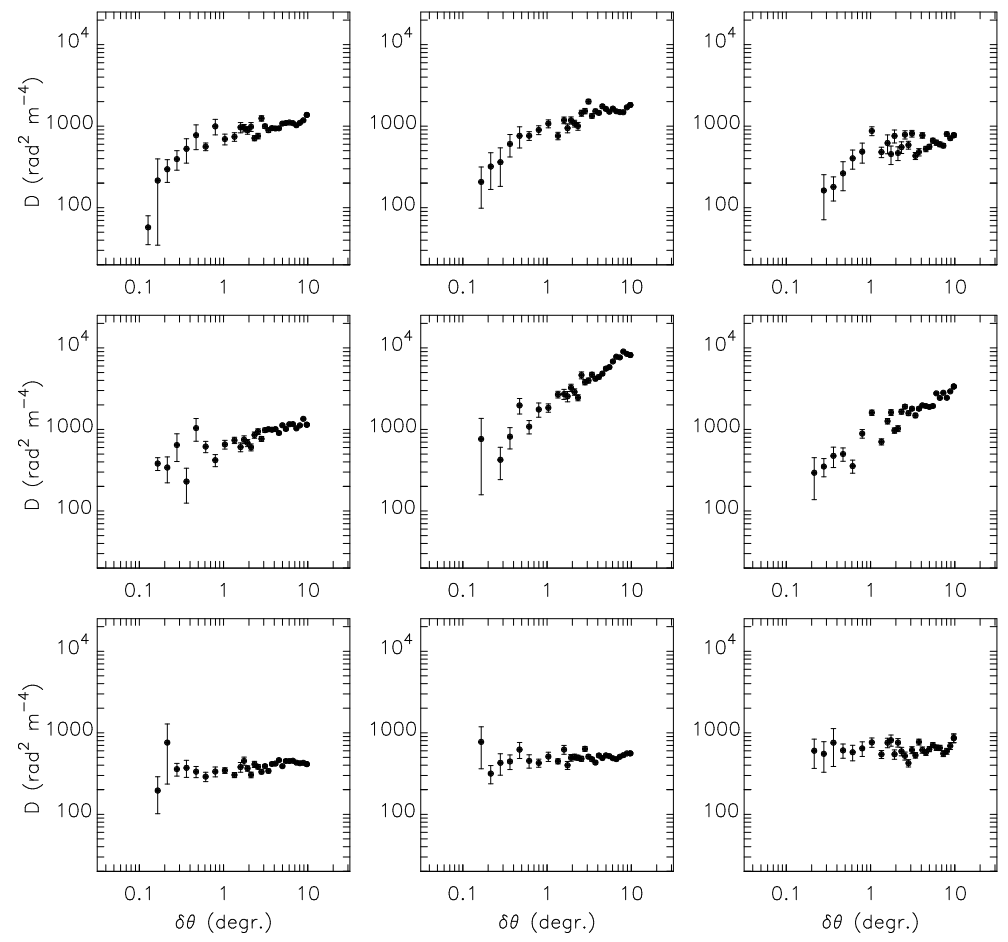

Figure 2: Sample structure functions showing a broken power law (top row), single power law (middle row) and essentially flat (bottom row) structure function.

probed by 20 RMs with 5000 RMs per square degree (approximately an SKA all-sky RM survey) and 15000 RMs per square degree (approximately an SKA deep polarization survey). An all-sky survey with the SKA will be able to probe magnetic fields in $\sim 1000$ galaxies, while a deep field of 40 square degrees will probe only a few galaxies with more than 20 background RMs per galaxy. Sample size is optimized by a shallow wide survey in this case, but magnetic field structure from background RMs can be derived only for local galaxies (distance less than $\sim 30 \mathrm{Mpc}$ ).

Information on magnetic fields in galaxies at high redshift comes from chance alignments of galaxies in the line of sight to a distant quasar. The association of excess RM toward quasars with strong Mg II absorption lines at a redshift smaller than the redshift of the quasar indicates the existence of large-scale magnetic fields in intervening galaxies [33, 34]. Since the galaxy itself is not observed directly, it is not known where the line of sight intersects the foreground galaxy. With only a single RM per galaxy, there is no information on the structure of the magnetic field, besides the fact that it has a large-scale component.

Tracing the evolution of magnetic field structure in galaxies over most of the age of the universe requires observations of synchrotron emission of galaxies in a polarization deep field. Today, information on magnetic field structure of galaxies outside the Local Group comes from imaging of polarized synchrotron emission. A review of the state of observations in this area was given by []. Future telescopes will similarly extend observations of magnetic field structure to more distant galaxies that cannot be probed with a sufficient number of background RMs. However, imaging polarimetry of normal disk galaxies at redshift $1-2$ with angular resolution $\sim 0.1^{\prime \prime}$ necessary to resolve the disk is well beyond the sensitivity limit of even the SKA. 

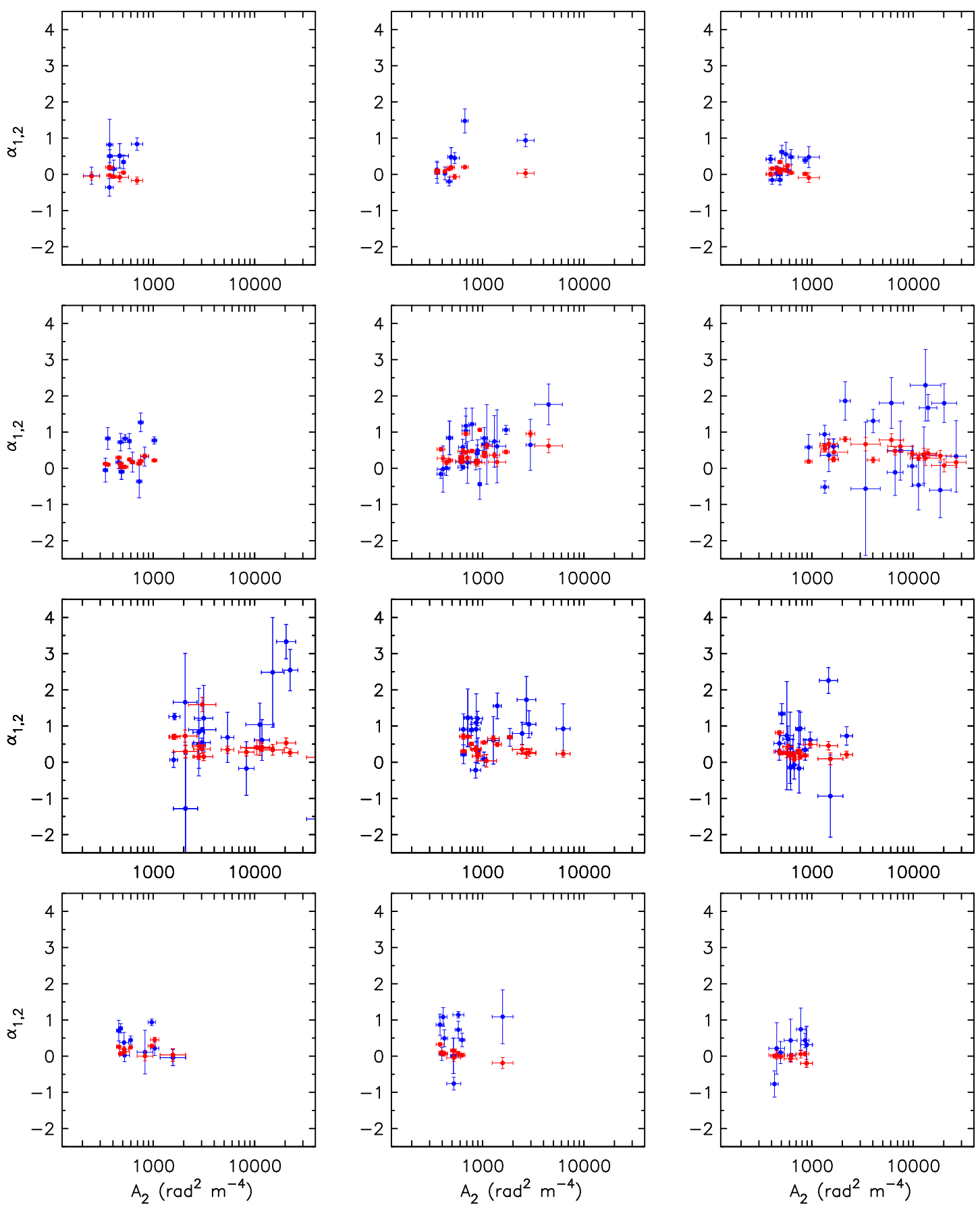

Figure 3: Slope versus amplitude RM for structure functions in independent areas of the sky, each approximately 15 degrees on a side. Each panel shows fields with the same Galactic latitude, ranging from the North Galactic Pole (upper left panel) to the South Galactic Pole (lower right panel) in reading order. Blue symbols represent power law fits to the SF for $\delta \theta<1^{\circ}$, and red symbols represent fits for $\delta \theta>1^{\circ}$. 


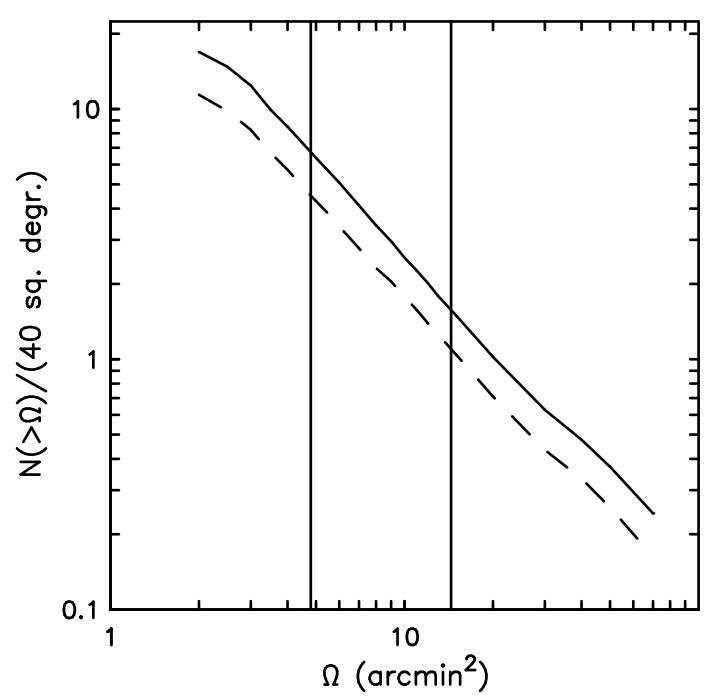

Figure 4: Number of galaxies in a 40 square degree field with with solid angle larger than $\Omega$, where galaxy solid angle is the area within 2 optical radii as listed in the UGC [32]. The dashed curve represents spiral galaxies, the solid curve all UGC galaxies. The two vertical lines mark the solid angles where galaxies are probed by at least 20 background RMs assuming 5000 RMs per square degree (right; SKA wide area survey) and 15000 RMs per square degree (left; SKA deep field).

However, polarization of unresolved normal disk galaxies can also be used [35, 36]. The degree of polarization of a spiral galaxy contains information on the uniformity of its magnetic field, in particular at higher frequencies (e.g. $4.8 \mathrm{GHz}$ ) where models of the integrated polarization indicate that uniformity of the magnetic field and inclination are the most important parameters for galaxies with inclination $<50^{\circ}$ [35]. This allows statistics of the uniformity of magnetic fields in spiral galaxies to be derived in a resolution-independent way. It is feasible to observe significant samples of galaxies in the local universe with a large single dish radio telescope. These observations can serve as a local comparison sample for observations of more distant spiral galaxies at the same rest frame wavelength to trace the evolution of magnetic fields in spiral galaxies.

\section{References}

[1] Han, J. L., Manchester, R. N., Lyne, A. G., Qiao, G. J., \& Van Straten, W. 2006, ApJ, 642, 868

[2] Brown, J. C., Haverkorn, M., Gaensler, B. M., Taylor, A. R., Bizunok, N. S., McClure-Griffiths, N. M., Dickey, J. M., \& Green, A. J. 2007, ApJ 663, 258

[3] Men, H., Ferrière, K., Han, J. L. 2008, A\&A, 486, 819

[4] Beck, R. 2009, this meeting

[5] Heald, G., Braun, R., \& Edmonds, R. 2009, A\&A, 503, 409

[6] Simard-Normandin, M., \& Kronberg, P. P. 1979, Nat. 279, 115

[7] Simard-Normandin, M., \& Kronberg, P. P. 1980, ApJ, 242, 74

[8] Beck, R. \& Gaensler, B. M. 2004, Observations of magnetic fields in the Milky Way and in near by galaxies with a Square Kilometre Array, in New Astronomy Reviews, 48, 1289 
[9] Stepanov, R., Arshakian, T. G., Beck, R., Frick, P., \& Krause, M. 2008, A\&A, 480, 45

[10] Taylor, A. R., Stil, J. M., Grant, J. K., Landecker, T. L., Kothes, R., Reid, R., Gray, A. D., Scott, D., Martin, P. G., Boothroyd, A. I., Joncas, G., Lockman, F. J., English, J., Sajina, A., \& Bond, J. R. 2007, ApJ, 666, 201

[11] Stil, J. M., Taylor, A. R., Krause, M., \& Beck, R. 2007, PoS(MRU)069 arXiv : 0802.1374

[12] O’Sullivan, S. P., Stil, J. M., Taylor, A. R., Ricci, R., Grant, J. K., \& Shorten, K. Proceedings of The 9th European VLBI Network Symposium on The role of VLBI in the Golden Age for Radio Astronomy and EVN Users Meeting, September 23-26, 2008, Bologna, Italy, arXiv : 0902.1995

[13] Taylor, A. R., Stil, J. M., \& Sunstrum, C. 2009, ApJ, 702, 1230

[14] Finkbeiner, D. 2003, ApJS, 146, 407

[15] Mitra, D., Wielebinski, R., Kramer, M., \& Jessner, A. 2003, A\&A, 398, 993

[16] Simard-Normandin, M., \& Kronberg, P. P. 1980, ApJ, 242, 74

[17] Snowden, S. L., Egger, R., Freyberg, M. J., McCammon, D., Plucinsky, P. P., Sanders, W. T., Schmitt, J. H. M. M., Trümper, J., \& Voges, W. 1997, ApJ, 485, 125

[18] Snowden, S. L., Egger, R., Finkbeiner, D. P., Freyberg, M. J., \& Plucinsky, P. P. 1998, ApJ, 493, 715

[19] Xu, Y., Kronberg, P. P., Habib, S., \& Dufton, Q. W. 2006, ApJ 637, 19

[20] Simonetti, J. H., Cordes, J. M., \& Spangler, S. R. 1984, ApJ, 284, 126

[21] Simonetti, J. H., Cordes, J. M. 1986, ApJ, 310, 160

[22] Minter, A. H., \& Spangler, S. R. 1996, ApJ, 458, 19

[23] Stil, J. M., Taylor, A. R., \& Sunstrum, C. 2009, in prep.

[24] Haverkorn, M., Katgert, P., \& De Bruyn, A. G. 2003, A\&A, 403, 1045

[25] Sun, X. H., \& Han, J. L. 2004, in The Magnetized Interstellar Medium, Proceedings of the conference, held in Antalya, Turkey, September 8 - 12, 2003, Eds: B. Uyaniker, W. Reich, and R. Wielebinski, Copernicus GmbH, Katlenburg-Lindau., 25

[26] Haverkorn, M., Gaensler, B., Brown, J. C., Bizunok, N. S., McClure-Griffiths, N. M., Dickey, J. M., \& Green, A. J. 2006, ApJ 637, L33

[27] Roy, S., Rao, A. P., \& Subrahmanyan, R. 2008, A\&A, 478, 435

[28] Haverkorn, M., Brown, J. C., Gaensler, B. M., \& McClure-Griffiths, N. M. 2008, ApJ, 680, L362

[29] Han, J. L., Beck, R., \& Berkhuijsen, E. M. 1998, A\&A, 335, 1117

[30] Gaensler, B., Haverkorn, M., Staveley-Smith, L., Dickey, J. M., McClure-Griffith, N. M., Dickel, J. R., \& Wolleben, M. 2005, Science, 307, 1610

[31] Mao, S. A., Gaensler, B. M., Stanimirović, S., Haverkorn, M., McClure-Griffith, N. M., Staveley-Smith, L., \& Dickey J. M. 2008, ApJ, 688, 1029

[32] Nilson, P. 1973, Uppsala General Catalogue of Galaxies (Uppsala: Uppsala Astron. Obs.) (UGC)

[33] Bernet, M., Miniati, F., Lilly, S. J., Kronberg, P. P., \& Dessauges-Zavadsky, M. 2008, Nature, 454, 302

[34] Kronberg, P., P., Bernet, M. L., Miniati, F., Lilly, S. J., Short, M. B., \& Higdon, D. M. 2008, ApJ, 676, 70

[35] Stil, J. M., Krause, M., Beck, R., \& Taylor, A. R. 2009, ApJ, 693, 1392

[36] Stil, J. M., Krause, M., Mitchell, L., Beck, R., \& Taylor, A. R. 2009, IAUS, 259, 543 